\title{
El sitio arqueológico de La Chincúa y la ofrenda del Plan de la Mesita, Chiapas
}

\section{La Chincúa Archaeological Site and the Offering of Plan de la Mesita, Chiapas}

\author{
Carlos Navarrete Cáceres \\ Instituto de Investigaciones Antropológicas \\ Universidad Nacional Autónoma de México, México
}

\begin{abstract}
Resumen: El conjunto arqueológico La Chincúa-Plan de la Mesita forma parte del complejo de sitios agrupados alrededor del Cerro Bernal, eminencia que domina la sección intermontana más angosta de la ruta costera que atravesaba el Soconusco chiapaneco rumbo a Guatemala. La ocupación del sitio La Chincúa inicia en el Preclásico Tardío, acoge durante el Clásico influencias teotihuacanas y continúa vigente durante el Posclásico como centro habitacional y religioso. Durante esta última época el vecino sitio de Plan de la Mesita se convirtió en lugar de enterramiento de personajes de élite y es de allí de donde proviene la ofrenda de objetos asociados a manufacturas textiles que aquí se analizan.
\end{abstract}

Palabras Clave: Soconusco; Chiapas; artefactos de madera; textiles; cronología.

ABSTRACT: The archaeological group La Chincúa-Plan de la Mesita is part of a complex of sites concentrated around Cerro Bernal, a hill that dominates the narrowest intermountain section of the coastal route from Chiapas toward Guatemala across the Soconusco. La Chincúa's settlement begins during the Late Preclassic, incorporates Teotihuacan influence during the Classic, and continues on as a religious and residential center during the Postclassic. It is during this last period that La Chincúa's neighboring site, Plan de la Mesita, becomes an elite burial ground; the textile manufacturing objects offering here analyzed comes from this context.

KEYworDS: Soconusco; Chiapas; wooden artifacts; textiles, chronology.

RECEPCIÓN: 18 de marzo de 2020.

ACEPTACIÓN: 19 de mayo de 2020.

Dor: https://doi.org/10.19130/iifl.ecm.57.2021.18651 
El sitio arqueológico de La Chincúa se encuentra en el kilómetro 27 de la carretera costera Tonalá-Tapachula, cuyo trazo irrumpió en el sitio destruyendo parte de la sección central de donde se extrajo material de balastre; por otra parte, las siembras y quemas anuales han contribuido a dañar la arquitectura, sobre todo del área habitacional.

La Chincúa forma parte del conjunto de sitios agrupados alrededor del Cerro Bernal (Navarrete, 1966), el cual destaca por su altura y por el impresionante peñón que le da nombre, alcanzando los $1050 \mathrm{msnm}$ (Figura 1). Situado en un valle intermontano entre éste y el Cerro del Pato o Plan de la Mesita, constituye la parte más estrecha del camino costero que desde época prehispánica se dirigía al istmo centroamericano (Figuras 2 y 3), un punto geográfico propicio para el control de la ruta y paso obligado de los pochteca en el camino mexica hacia Ayotla, actual Tecún Umán, Guatemala, población de filiación maya (Chapman, 1959). Una razón geográfica para que cientos de años después se diera aquí la batalla más al sur entre el ejército insurgente y el realista durante la guerra de Independencia (López Vasallo, 2012).

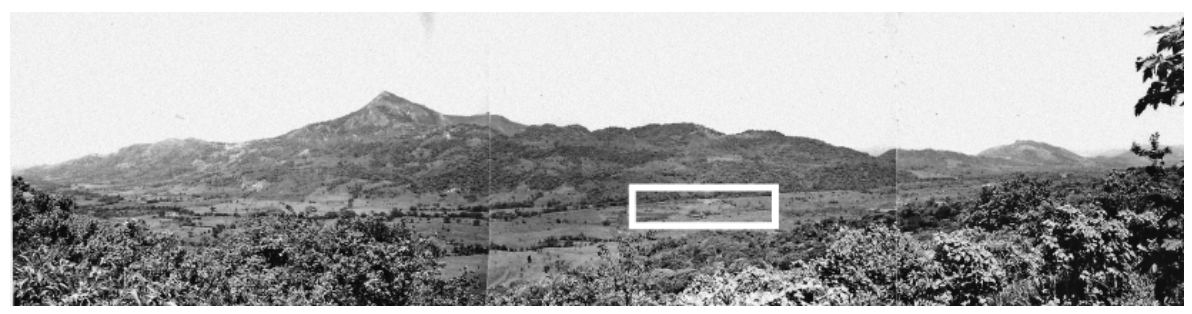

Figura 1. El Cerro Bernal y el Valle de La Chincúa, desde el plan de La Mesita. Se remarca la ubicación del sitio arqueológico (Fotografía: C. Navarrete).

\section{El sitio arqueológico}

La siguiente descripción fue hecha en 1968, a partir del reconocimiento de superficie desarrollado en la costa de Chiapas por el autor. ${ }^{1}$ Desde entonces el sitio ha sufrido una sistemática destrucción y desconocemos su estado actual. En ese tiempo se conservaban cinco montículos relativamente completos, con alturas de entre 3 y $5 \mathrm{~m}$, todos dentro de una conformación general a base de amplias terrazas (Figura 4).

${ }^{1}$ El recorrido arqueológico en la costa de Chiapas $(1968,1973$ y 1985) fue patrocinado por la Fundación Arqueológica del Nuevo Mundo, Brigham Young University (en adelante NWAF, BYU) en colaboración con el Instituto de Investigaciones Antropológicas de la Universidad Nacional Autónoma de México. 


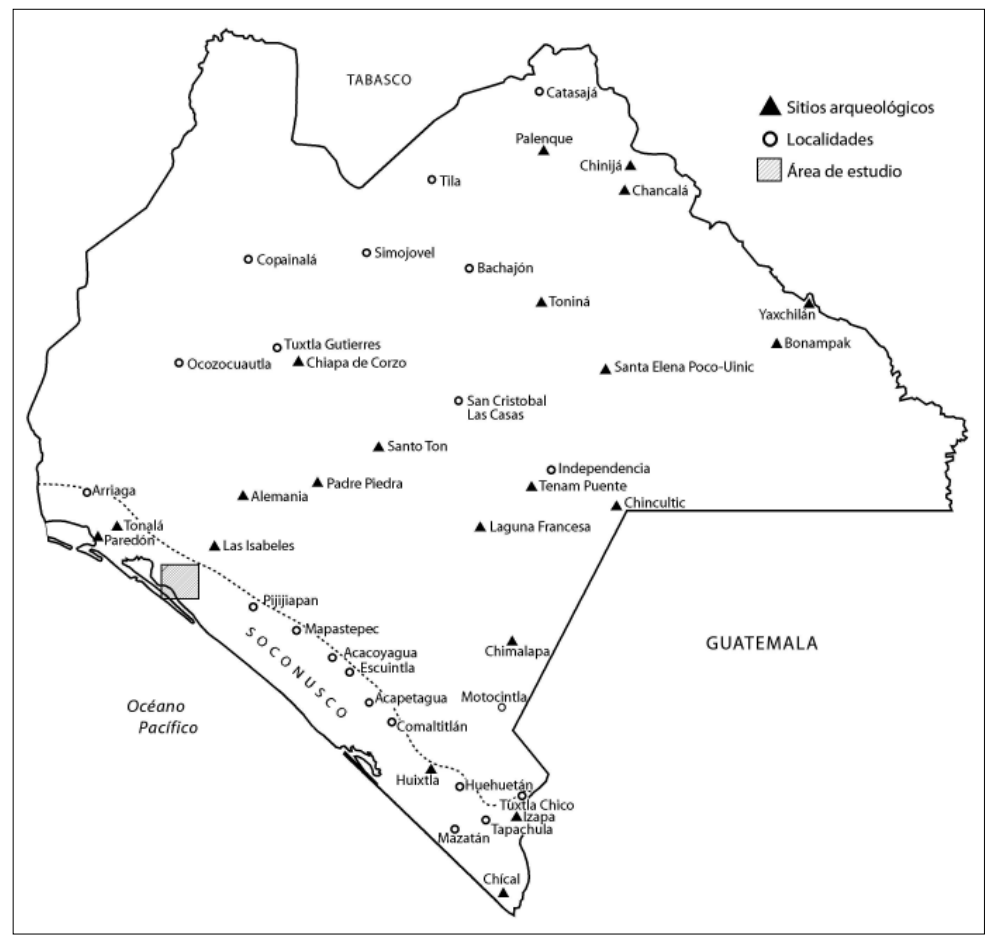

Figura 2. El estado de Chiapas y algunos puntos de referencia. Se remarca el área de estudio (Dibujo IIA, UNAM).

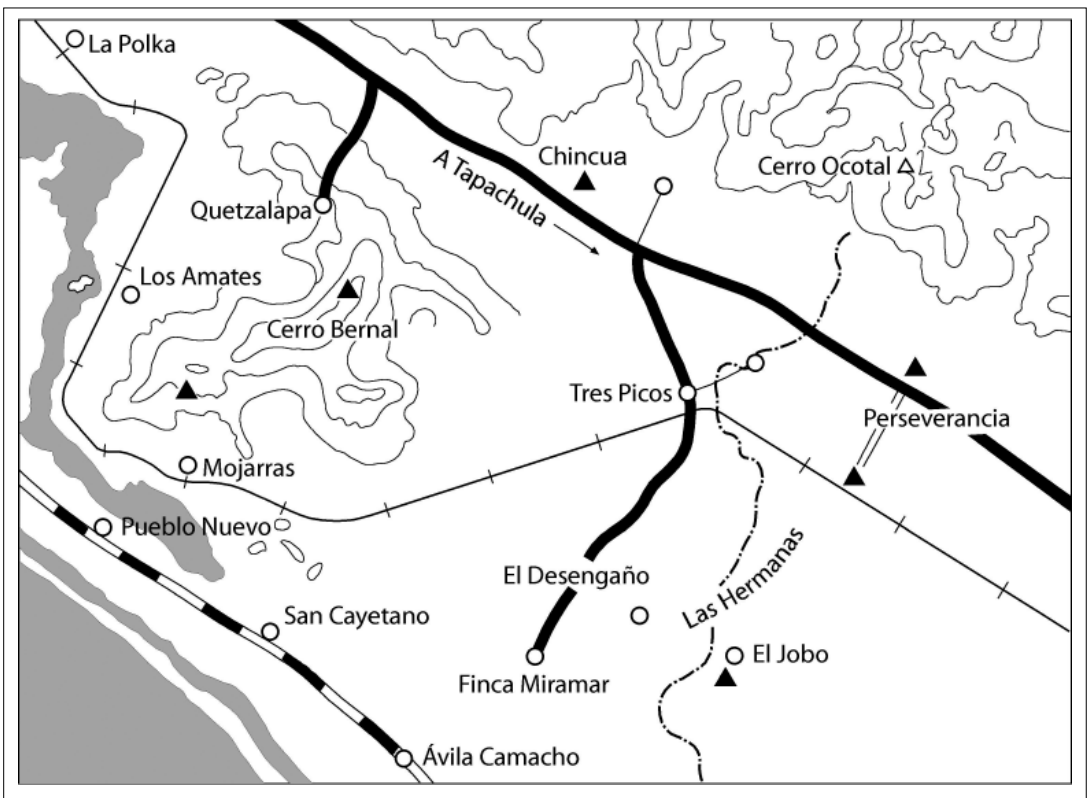

Figura 3. La Chincúa y otros sitios arqueológicos del entorno (Dibujo IIA, UNAM). 


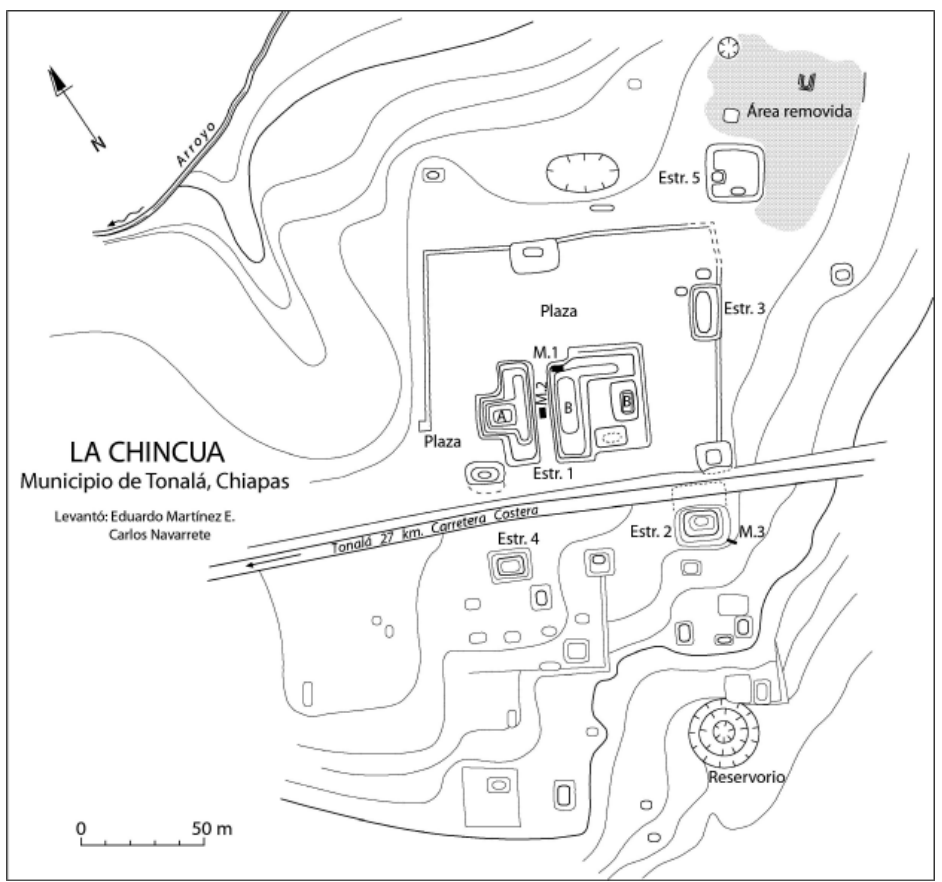

Figura 4. Plano general de La Chincúa, con base en los reconocimientos de 1973 y 1985 (Levantamiento de Eduardo Martínez E. y Carlos Navarrete).

El conjunto mejor conservado es el de la cancha de juego de pelota, asentado sobre una plaza de aproximadamente $1.20 \mathrm{~m}$ de altura por $100 \mathrm{~m}$ de lado. Se compone de las dos banquetas tradicionales, sin cabezales, asociada con otras construcciones: la Banqueta $\mathrm{A}$, con un basamento piramidal, y la Banqueta $\mathrm{B}$, con un patio delimitado por dos plataformas bajas. Dos montículos con huellas de saqueo se orillan en la parte libre de la plaza. El sistema de construcción es a base de un núcleo de tierra revuelta con piedras pequeñas y cantos rodados, recubierto de sillares burdamente careados y un aplanado exterior de barro. La orientación general es al $\mathrm{N} 25^{\circ} \mathrm{E}$.

\section{Los monumentos esculpidos}

Son tres piezas, dos de ellas localizadas en las instalaciones de la cancha de pelota, sumamente intemperizadas.

Monumento 1. Marcador del juego de pelota; mide $1.47 \mathrm{~m}$ por $65 \mathrm{~cm}$ de ancho y $21 \mathrm{~cm}$ de grueso (Figura 5a). Representa una cabeza de guacamaya o serpiente con su respectiva espiga. Debió de haber estado al centro de la Plataforma 
A de la cancha, pero con la construcción de la carretera alteraron secciones del conjunto y el marcador fue movido de lugar.

Monumento 2. Estela de $1.93 \mathrm{~m}$ de largo por $85 \mathrm{~cm}$ de ancho y $20 \mathrm{~cm}$ de grueso (Figura 5b). Los motivos originales se encuentran borrados debido al reúso de la pieza como altar durante la última época de ocupación, en la que prácticamente cubrieron la superficie con pequeños cuencos en función de algún tipo de oblaciones. Permanecía en el interior de la sección central de la cancha, botada boca arriba.

Monumento 3. Laja recortada a los lados, sumamente intemperizada y golpeada (Figura 5c); aún así, se nota que los rasgos fueron esculpidos sin mayor cuidado. Mide $1.40 \mathrm{~m}$ de largo y $15 \mathrm{~cm}$ de grueso. Se percibe el rostro de un personaje con el brazo derecho hacia abajo y señalada la línea de separación de las piernas. Estaba tirado al pie de la Estructura 2, evidentemente movido de lugar.
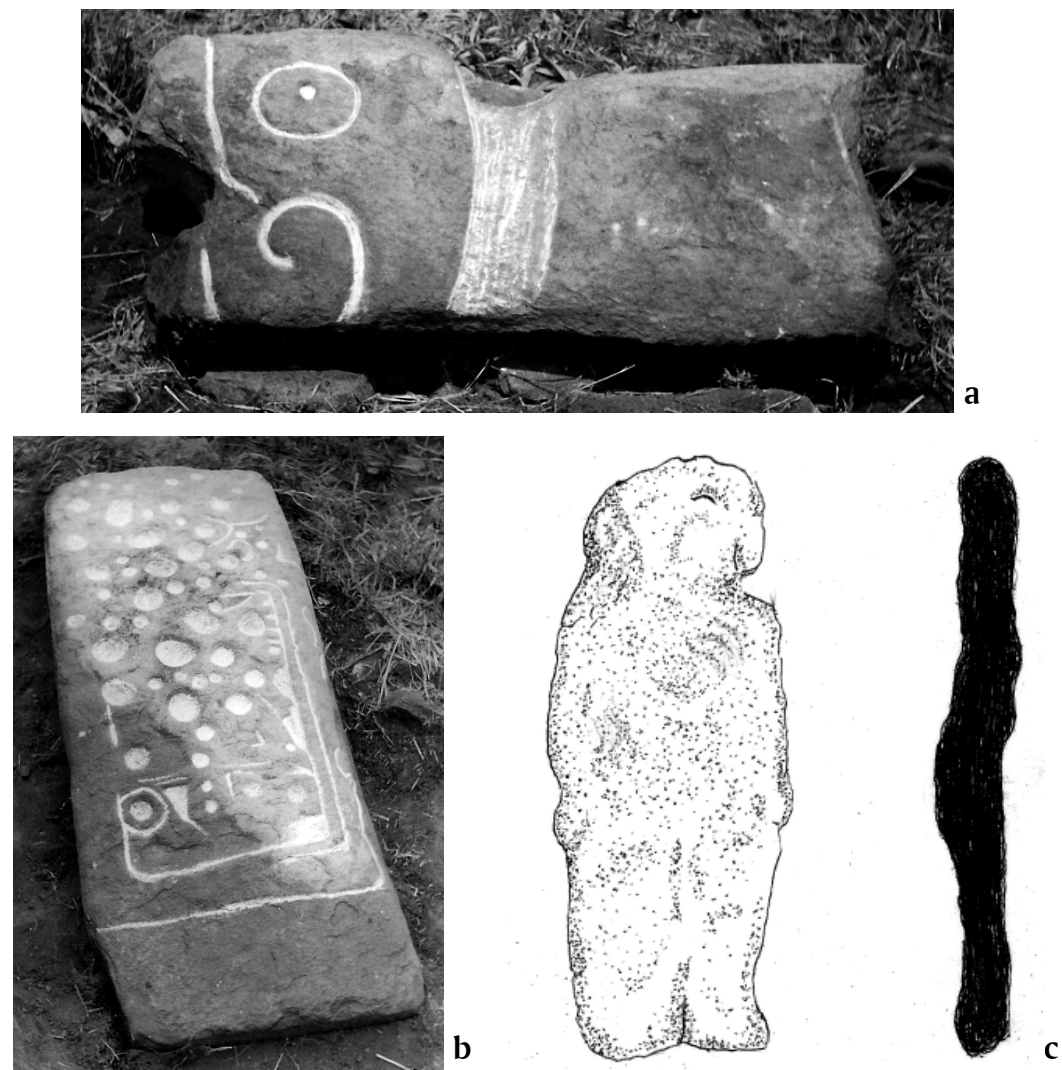

Figura 5. a) Monumento 1: Marcador del juego de pelota con la representación de una cabeza de guacamaya o serpiente; b) Monumento 2: Estela reusada por medio de pequeños cuencos que borraron el motivo original; c) Monumento 3: Figura esculpida en una laja caliza, destruida quizá intencionalmente (Fotografías y dibujo: C. Navarrete). 


\section{Cerámica}

La muestra recolectada en superficie aportó muestras del Preclásico Superior, el Clásico y numerosas evidencias de ocupación a lo largo del Posclásico. ${ }^{2}$ Como ejemplo exponemos algunos ejemplares de perfiles tempranos consistentes en platos de bordes hacia afuera y fondo plano (Figuras 6a-c), semejantes a formas correspondientes a tipos color rojo pulido de la fase Guillén de Izapa (Lowe, Lee y Martínez, 2000) y de formas de cerámica local de inspiración teotihuacana: vasos de paredes altas, con soportes cuadrados y decoración esgrafiada (Figuras $6 \mathrm{~d}$ y e). La presencia teotihuacana en la región - cerámica y monumentos esculpidos - ha sido documentada en el vecino sitio de Los Horcones, en la parte alta del Cerro Bernal (Navarrete, 1966: 23-45).

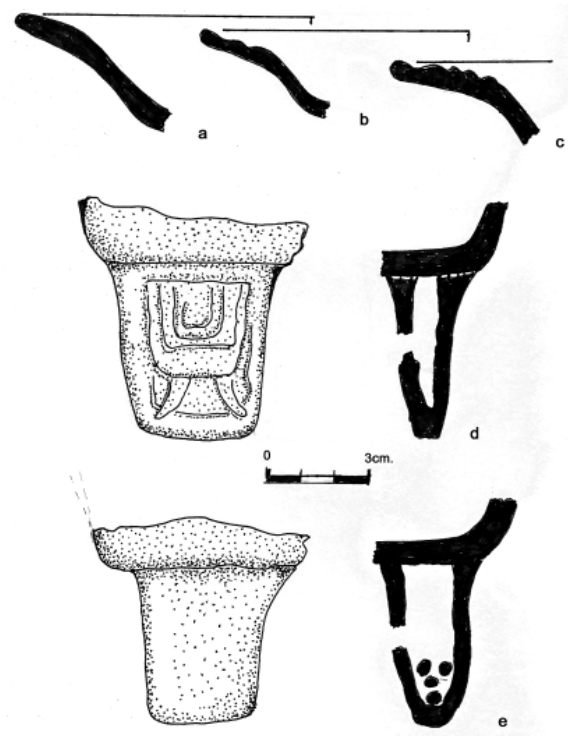

Figura 6. Cerámica: a-c) Platos del Preclásico Superior de borde hacia fuera y color rojo pulido; $\mathrm{d}$, e) Soportes de influencia teotihuacana de factura local (Dibujo: C. Navarrete).

\section{El Plan de la Mesita}

Enfrente de La Chincúa se levanta el Cerro del Pato o Plan de la Mesita, perteneciente a la serranía de El Ocotal, en donde se encuentra la pintura rupestre que da nombre al sitio. El sendero de ascenso para acceder a la cima comienza en el

\footnotetext{
${ }^{2}$ El análisis del material cerámico será publicado en un volumen que abarcará las investigaciones arqueológicas a lo largo de la costa del Pacífico (Navarrete, en proceso).
} 
rancho Reforma, en un trayecto que dura cerca de hora y media. El terreno que lo corona y en el que se disponen las evidencias arqueológicas forma una especie de hamaca en cuyos extremos emergen dos formaciones rocosas (Figuras 7a y b). De un lado tres rocas dan la impresión de ser estelas naturales, y en el opuesto, otro
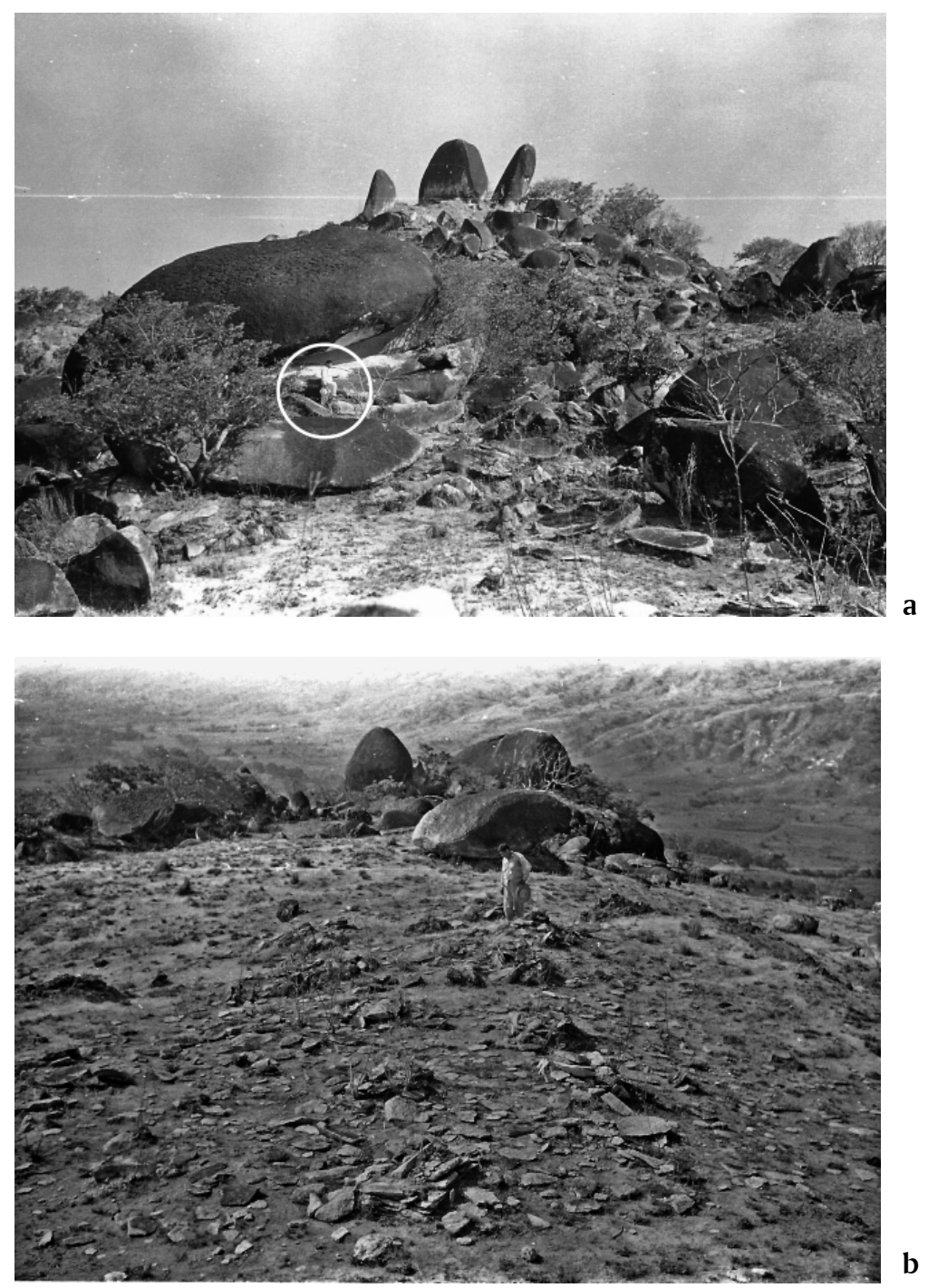

Figura 7. El Plan de la Mesita y las rocas-estelas: a) "Piedra del pato”, el círculo señala la ubicación de la pintura rupestre; b) Las rocas del lado opuesto y el área de entierros (Fotografías: C. Navarrete). 
conjunto alberga una roca redondeada con un nicho natural, en cuyo interior se encuentran dos diseños pintados en color rojo cinabrio de $20 \mathrm{~cm}$ de largo, con la representación de una mano y un ave parecida a un pato (Figura 8).

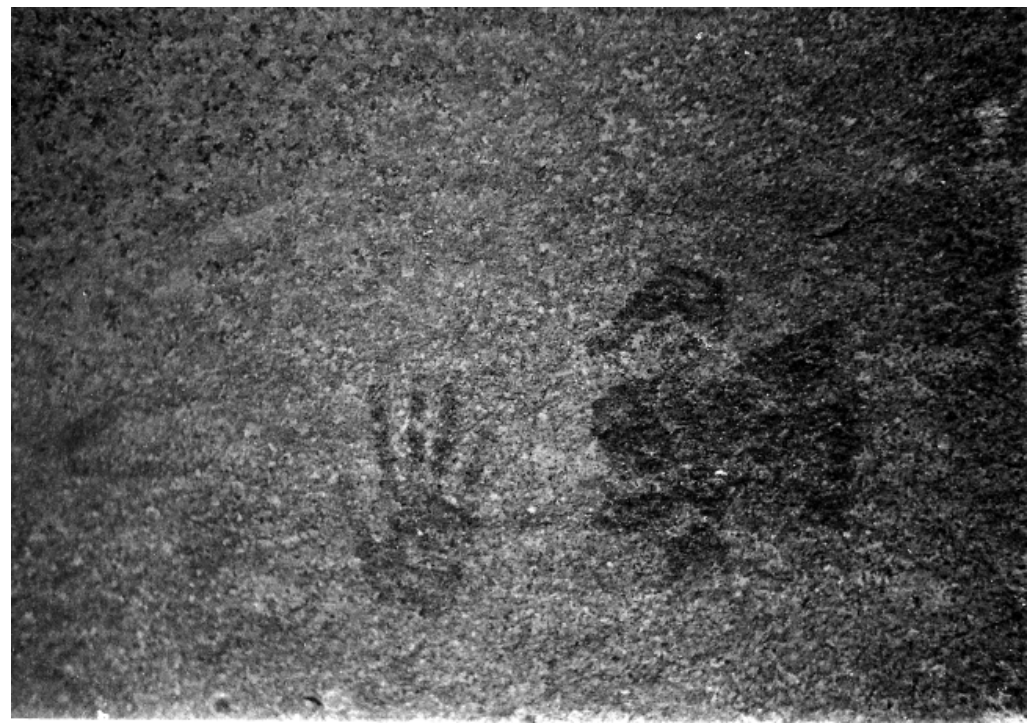

Figura 8. La pintura del pato y la impresión de una mano, en color rojo cinabrio (Fotografía: C. Navarrete).

A unos 25 metros del primer conjunto se encuentran catorce pequeños cuadros formados por pequeñas lajas dispuestas en tres filas, orientadas en un eje $\mathrm{N} 10^{\circ} \mathrm{W}$ entre los dos conjuntos de rocas. Miden un promedio de $40 \mathrm{~cm}$ de lado y $30 \mathrm{~cm}$ de altura (Figura 9). De acuerdo con rumores de actividades de saqueo, cada uno señalaría el entierro de grandes ollas que contenían restos humanos, "como si estuvieran sentaditos". Los fragmentos de estas urnas funerarias dispersas en el entorno se equiparan formalmente con una pieza completa procedente del lugar, perteneciente a la colección del doctor Óscar Rueda Escobar en la ciudad de Tonalá (Figura 10). Obviamente se trataría de un antiguo cementerio, en el cual contamos tres sepulcros completos y once saqueados. De uno de ellos proviene la ofrenda a la que nos referiremos a continuación.

\section{La ofrenda del Plan de la Mesita}

En 1965 el citado doctor Rueda recibió, como obsequio de unos campesinos, pacientes suyos, un conjunto de objetos, la mayoría de madera. Sobre la procedencia, le informaron que, habiendo oído que en el Cerro del Pato, debajo de las "pilitas" de piedra había tesoros, la curiosidad los llevó a excavar una, de la que 


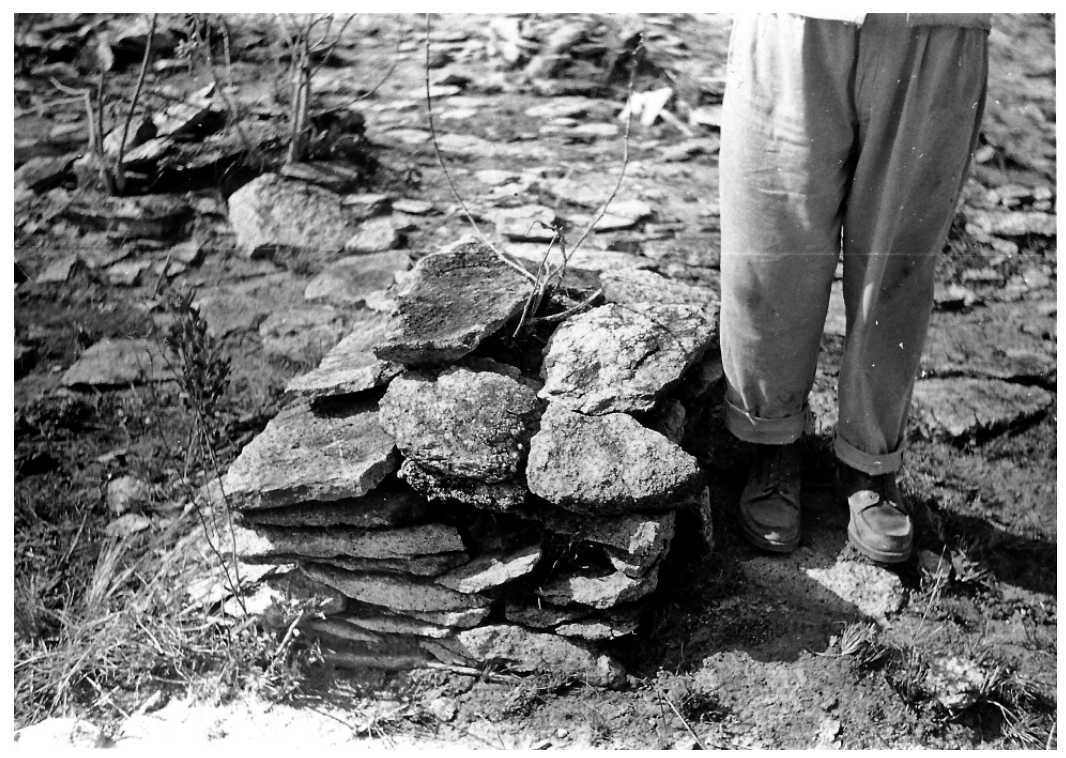

Figura 9. Acercamiento al área de entierros, señalados por cuadros formados de lajas cortadas (Fotografía: C. Navarrete).

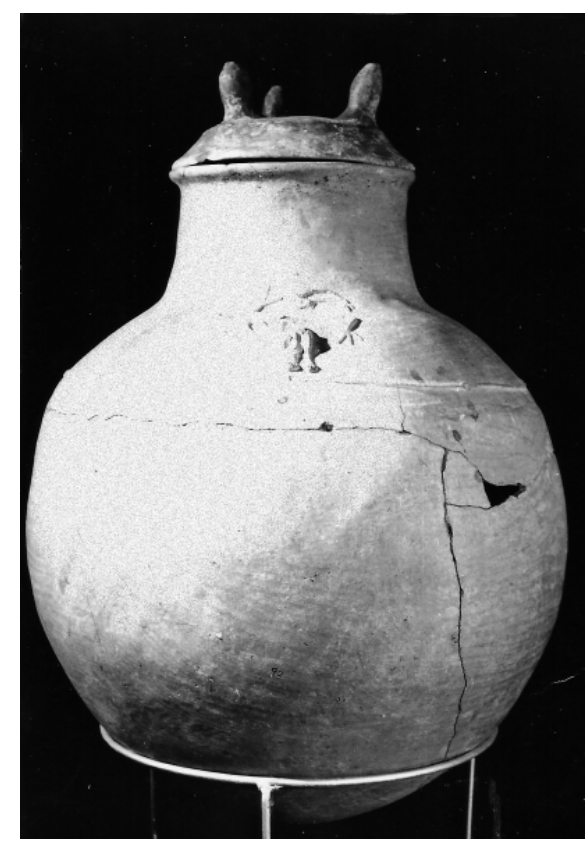

Figura 10. Urna funeraria con la boca tapada por una vasija trípode de época posclásica. Colección Óscar Rueda Escobar, Tonalá (Fotografía: Mario Vega, NWAF, BYU). 
removieron una olla grande rota de arriba, donde sacaron "unos huesos y una tela" que se deshicieron con el aire, lo que atribuyeron a que "tenían encanto"; únicamente los objetos de madera se conservaron. El doctor encargó a un artista plástico de Tonalá su restauración, y las piezas fueron sumergidas en parafina líquida para estabilizarlas; tiempo después fueron donadas al Instituto Nacional de Antropología e Historia, a través del entonces Departamento de Prehistoria, en cuyos laboratorios recibieron un tratamiento adecuado. Actualmente, la mayoría se encuentra en exhibición en el Museo Regional de Chiapas, en Tuxtla Gutiérrez. Tres de ellas se extraviaron en el trayecto.

En la siguiente lista se ofrecen las principales características de cada pieza, con el respectivo número del inventario oficial. Los nombres que reciben en Chiapas de acuerdo con su función fueron tomados del estudio de los textiles zoques de Cordry y Cordry (1988: 147-161); los equivalentes en el centro de México de García Vargas (1975), y los de la costa chica de Oaxaca de Ryesky (1975). ${ }^{3}$

1. Bobina o lanzadera. Mide $19 \mathrm{~cm}$ de largo y $1.8 \mathrm{~cm}$ de diámetro máximo. Decoración a base de pequeñas muescas triangulares. Herramienta fundamental para los telares de mano. No. de catálogo 10-219051 (Figura 11a).

2. Aguja de hueso. Mide $19 \mathrm{~cm}$ de largo. Decorada en el extremo de agarre con la representación de una mano, a su vez adornada con una pulsera decorada con líneas y círculos. No. de catálogo 10-219051 (Figura 11b).

3. Eje de huso. Mide $24.5 \mathrm{~cm}$ de largo. Aguzado de los dos extremos. No. de Catálogo 10-219053 (Figura 11c).

4. Separador de hilos. Mide $13.2 \mathrm{~cm}$ de largo y $0.9 \mathrm{~cm}$ de ancho. No. de catálogo 10-219055 (Figura 11d).
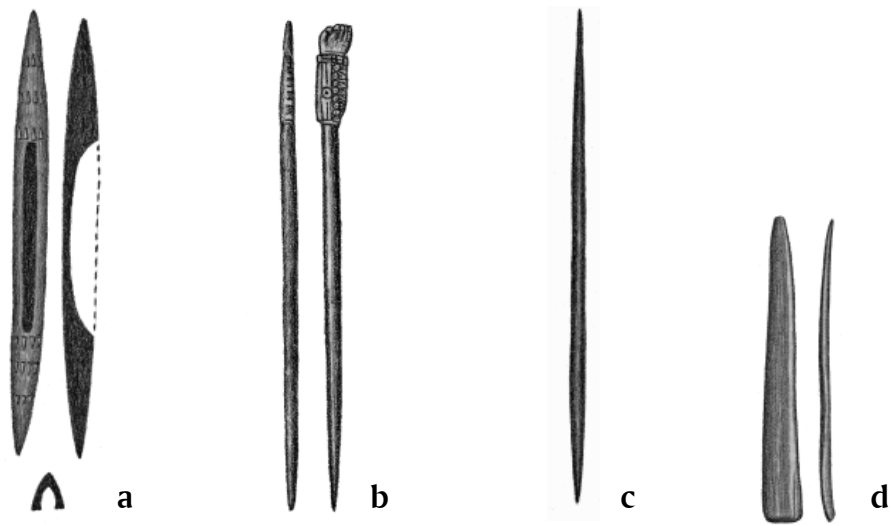

Figura 11. Artefactos de uso textil: a) bobina o lanzadera; b) aguja de hueso con la representación de una mano; c) eje de huso; d) separador de hilos (Dibujos de Ramírez Jiménez Pozo, nwaF, BYu).

\footnotetext{
${ }^{3}$ Los dibujos de las piezas fueron realizados por el artista chiapaneco Ramírez Jiménez Pozo (NWAF, BYU).
} 
5. Lanzadera con un extremo terminado en punta y el opuesto en dos apéndices separados, calados para funcionar como conductores de hilo en la urdimbre. No. de catálogo 10-219057 (Figura 12a).

6. Machete con la empuñadura labrada en forma de animal fantástico con la boca abierta; muestra colmillos, lleva orejeras y una cresta emplumada. Se desconoce su paradero (Figura 12b).

7. Machete terminado en punta. Mide $24.1 \mathrm{~cm}$ de largo y $2.2 \mathrm{~cm}$ de ancho. Representa un ave con el pico alargado. No. de catálogo 10-219058 (Figura 12c).
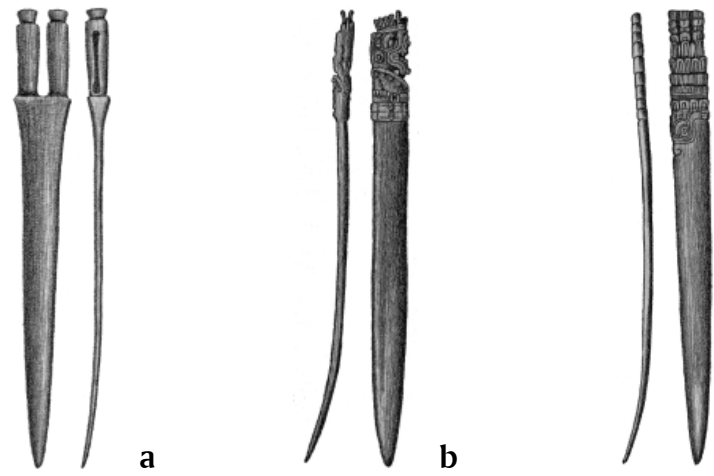

Figura 12. Artefactos especiales: a) lanzadera con un extremo calado para conducir el hilo de la urdimbre; b) machete con la empuñadura labrada en forma de animal fantástico;

c) machete en forma de ave con el pico alargado (Dibujos de Ramírez Jiménez Pozo, NWAF, BYu).

8. Cuatro rodillos, cruceras o varillas, tres de ellas con medidas de $16.5 \mathrm{~cm}$ de largo y un promedio de $9 \mathrm{~mm}$ de diámetro. Presentan ligeras variantes decorativas en los extremos, tres de ellas terminadas en un diseño común en forma de manojos de plumas. No. de catálogo 10-219060, 10-219061, 10-219062 (Figuras 13a, b, c); la cuarta mide $15.4 \mathrm{~cm}$ de largo y presenta un diseño semiredondo calado. No. de catálogo 10-219059 (Figura 13d).
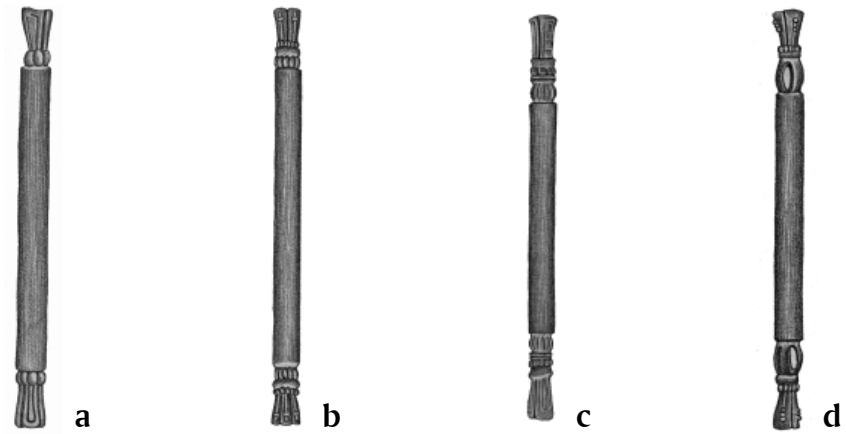

Figura 13. Cuatro rodillos, cruceras o varillas con ligeras variantes decorativas en los extremos, quizá flecos o plumas (Dibujos de Ramírez Jiménez Pozo, NWAF, BYu). 
9. Rodillo o enjulio de cabecera, decorado en los extremos con la figura de un tejón o andasolo y con una cabeza de animal no identificado. Es de mayor longitud que las demás (Figura 14). Se desconoce el paradero.
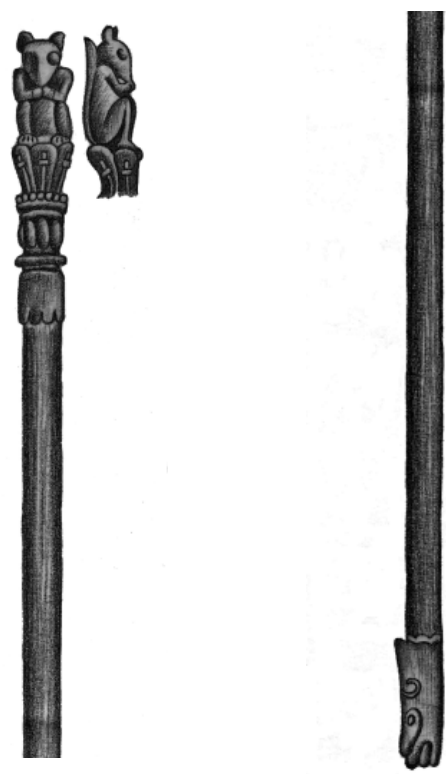

Figura 14. Rodillo o enjulio de cabecera, decorado en los extremos con figuras de animales. El tejón está sentado en un remate adornado con representaciones de plumas (Dibujos de Ramírez Jiménez Pozo, nWAF, BYu).

10. Peine de presión con mango. Empleado para afirmar la trama a medida que avanza el tejido. El ejemplar muestra el carácter de un objeto de ofrenda, no de uso práctico sino simbólico. El mango es de factura delicada, cubierto de un mosaico de pequeñas laminillas de piedra oscura pulida (Figura 15a). Se desconoce su paradero. Tratándose del área zoque, en el Tapesco del Diablo fue encontrada una peineta hecha con varillas aguzadas en los dos extremos, sujetas en el centro con un entrelace de cuerdas (Silva y Linares, 1993: foto 9), exactamente del mismo tipo de la referida etnográficamente por Cordry y Cordry (1988: 159).

11. Pasahilos con dos perforaciones; se buscó darle forma de cabeza de animal, no identificado. Mide $4.7 \mathrm{~cm}$ de largo y $2.7 \mathrm{~cm}$ de ancho. Parece fabricado de alguna semilla de consistencia dura. No. de catálogo 10-219052 (Figura 15b).

12. Dos malacates o contrapesos de huso. Hechos de barro cocido miden $2.5 \mathrm{~cm}$ de largo. No formaron parte del lote donado al Instituto Nacional de Antropología e Historia, y permanecieron en la colección Rueda de Tonalá (Figura 15c). Parecen ser las únicas piezas de uso práctico del lote, exactas a las que señala la etnografía moderna en el área zoque (Cordry y Cordry, 1988: 149). 


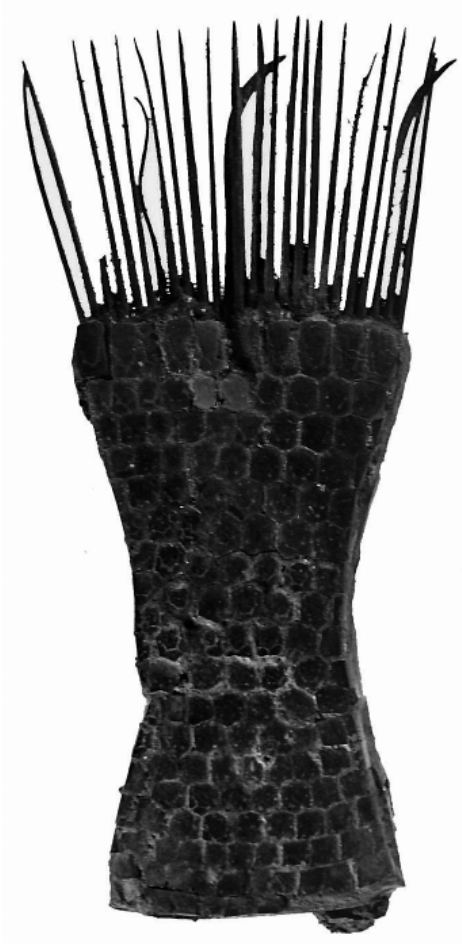

a

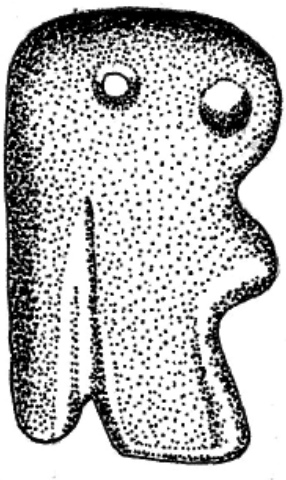

b

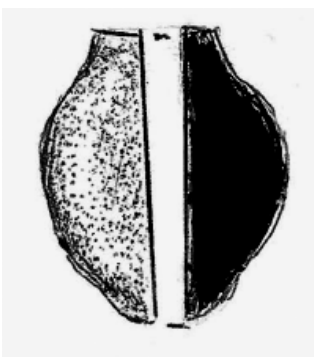

c

Figura 15. Tres instrumentos de uso textil: a) Peine de presión con el mango cubierto de laminillas de piedra pulida; b) Pasahilo con dos perforaciones en forma de cabeza de animal;

c) Malacate o cabeza de huso (Fotografía y dibujos: C. Navarrete).

\section{Consideraciones}

El hecho de tratar los sitios de La Chincúa y el Plan de la Mesita como una unidad se debe a la cercanía territorial que guardan y a que en la última época de ocupación compartieron los mismos materiales arqueológicos. En La Chincúa y sus alrededores se encuentran evidencias cerámicas del Preclásico Tardío y del Clásico Temprano, cosa que no ocurre en el Plan de la Mesita. Es en los dos momentos del Posclásico, Temprano y Tardío, que concuerdan, por ejemplo, en la presencia de tiestos de superficie del tipo Plumbate Tohil y de grandes urnas funerarias. Ya referimos que en la colección Rueda se conserva un ejemplar completo extraído de dicho cementerio, con la boca tapada por medio de una vasija trípode, evidentemente posclásica (Figura 10), y de La Chincúa proviene una urna de boca amplia color rojo ladrillo, decorada con la representación de una cabeza de murciélago (Figura 16). 


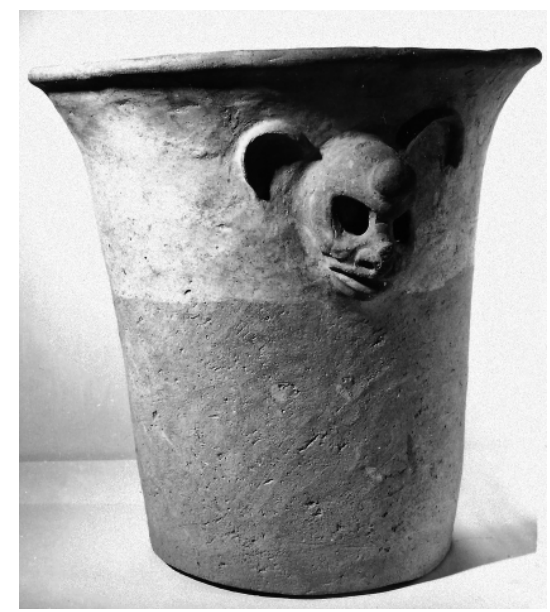

Figura 16. Urna funeraria con la representación de un murciélago. Procede de La Chincúa. Colección Óscar Rueda Escobar, Tonalá (Fotografía: C. Navarrete).

De la parte alta del cerro procede un plato trípode de soportes almenados cubierto de baño blanco, con diseños en color rojo (Figura 17a), y dos más con soportes zoomorfos fueron encontrados por agricultores en los alrededores de La Chincúa (Figuras 17b, c). Aunque desconocemos su contexto exacto constituyen pistas a seguir por su temporalidad tardía.
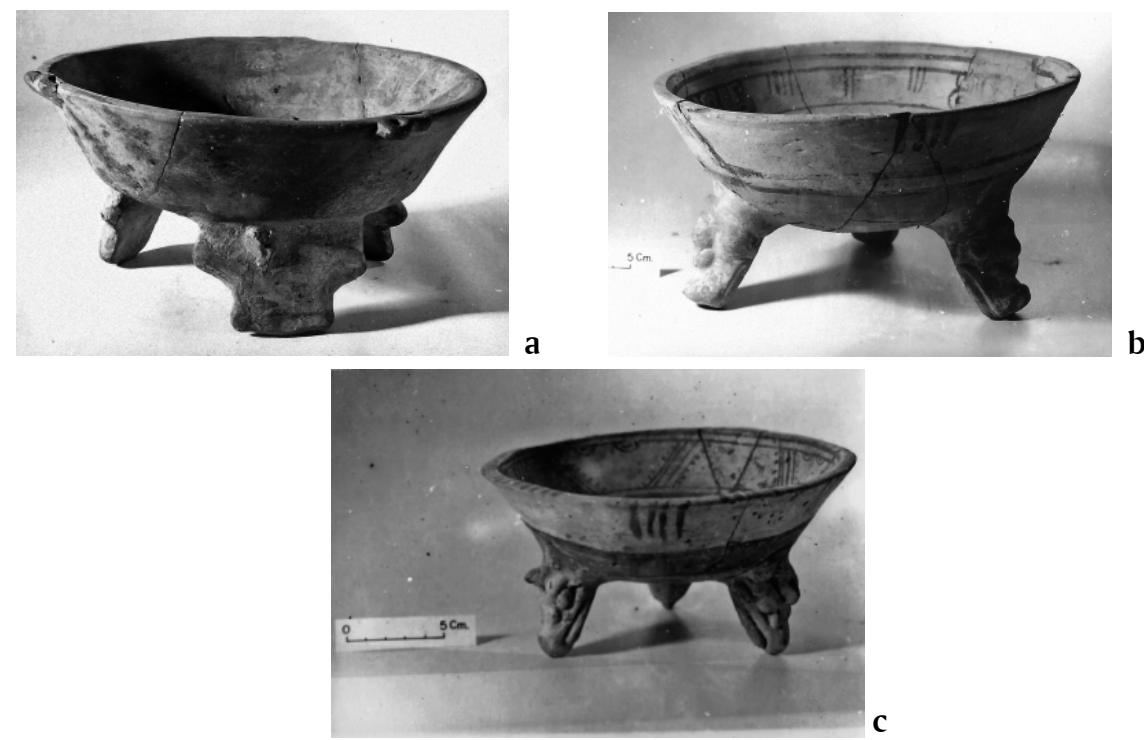

Figura 17. Tres vasijas trípodes de época posclásica: a) proviene del Plan de la Mesita; b-c) proceden de los alrededores de La Chincúa (Fotografías: C. Navarrete). 
El Monumento 1 es a todas luces un marcador del juego de pelota, y si la representación es de serpiente constituye una pieza más de una forma que se repite principalmente en el Posclásico. Si se trata de una guacamaya recordamos dos importantes ejemplos: los marcadores de Copán (Proskouriakoff, 1963: lám. 8) y los de Asunción Mita, en el oriente de Guatemala (Stromsvik, 1949); muy similar resulta también el marcador de guacamaya reportado recientemente del sitio Las Joyas de San Nicolás, ubicado en esta misma región guatemalteca, asociado al estilo Cotzumalguapa (Cossich et al., 2015: Fig. 3). El Monumento 2, cuyos diseños originales desaparecieron, es una estela reusada como altar al que cubrieron de pequeños cuencos, quizá durante la última época. Poco puede decirse del Monumento 3. En lo que se refiere a las pinturas rupestres, podría establecerse una comparación con la Piedra de Ayarza, en el oriente de Guatemala, donde se ha reportado la representación de un pato en color rojo ocre, que se ha relacionado con el estilo Mixteca-Puebla (Stone y Ericastilla, 1999: 686, 692), ejemplo que abonaría elementos a la discusión de un estilo pan-mesoamericano en esta región.

Las piezas de madera y hueso son representaciones ceremoniales de objetos para el trabajo textil. Seguramente constituyeron una ofrenda funeraria en forma de atado, a juzgar por la tela que se "hizo ceniza" en el proceso de saqueo. Sin función práctica, conllevan una intención funeraria que califica merecimiento y jerarquía. Una ofrenda de tal naturaleza se entiende en razón del respeto que merecían las tejedoras en época prehispánica, lo que en muchos sentidos continúa al presente (Pomar, 2005; Weitlaner, 2005), en el entendido de que había artesanas de telas de uso corriente para la gente común, y de telas de fino algodón con diseños especiales, prerrogativa de los sectores privilegiados (Weitlaner, 1959, 2005; Mastache, 2005). Quizá a este tipo pertenezcan las telas encontradas en las cuevas de Chiptic (Weitlaner, 1954), La Cieneguilla (O’Neale, 1942; Wauchope, 1942), La Garrafa (Landa, 1988), el Tapesco del Diablo (Silva y Linares, 1993; Linares y Silva, 2001) y la Cueva del Lazo (Domenici, 2009).

La cronología del conjunto puede establecerse por el estilo de la decoración de las piezas labradas de las Figuras 14a, 14b y 16, cuyos diseños se acercan al estilo pan-mesoamericano del Posclásico o "mixtecoide" para emplear una generalización.

Dada la importancia religiosa que tenían los afloramientos de rocas con determinadas formas y ubicación (Navarrete, 1991), resulta lógica la unidad que establecen los dos conjuntos pétreos, el cementerio y la pintura rupestre, que seguramente constituyó un elemento básico. Tomando en cuenta la antiguiedad que suele alcanzar esta manifestación artística, cabría la posibilidad de que, quienes decidieron ubicar en el lugar el cementerio, hubiesen encontrado la pintura realizada tiempo atrás, incorporándola a un nuevo ritualismo. Los extremos rocosos con formas de estelas naturales resultaron apropiadas para rendirle culto a la tierra, tal como sucede en otros sitios del área maya (Navarrete, 1991: 55), y la planada intermedia representó un punto adecuado en donde depositar los restos de personajes de élite, a juzgar por la calidad de las ofrendas. 
Al margen de la discusión sobre quiénes poblaron la franja costera del Soconusco en época temprana del Preclásico, por lo general atribuida a gente de filiación linguística mixe-zoqueana (Thompson, 1948; Coe, 1961), nuestro interés inmediato recae en la población que durante el Posclásico ocupó este territorio. Como remanentes de aquella primera ocupación habrá que atender el testimonio de la visita que fray Alonso Ponce hiciera a finales del siglo xvı a Tiltepec, población situada en el extremo occidental del Soconusco, en el que apuntó que "hablan una lengua que parece mucho a la zoque", y agrega que se hablaba una lengua parecida a la de Tuxtla, la cual no es otra que la zoque (Ciudad Real, 1976). Con datos ya del siglo xx, en el otro punto extremo del Soconusco, el filólogo Pablo González Casanova (1927) dio a conocer un vocabulario de la lengua que llamó tapachulteca II, de filiación mixeana (Wonderly, 1949).

Una corriente arqueológica abocada a los estudios en la costa de Chiapas propone una ocupación de gente de filiación linguística zoque-mixe con base en la información anterior (Lowe, Lee y Martínez, 2000); consideramos al respecto que hay que obrar con cautela y no generalizar con tan cortas evidencias, dada una realidad, en todo el territorio intermedio no se encuentra ni un solo término linguístico, ni topónimo alguno, que sea de raíz mixe-zoque, como acontece en la inmediata costa de Oaxaca. En cambio, es evidente la presencia de hablantes mayances; Culebro (1957: 6-7) reportó el mam en el área central —personalmente tuvo contacto con pequeños grupos asentados entre Acacoyagua y Huixtla- $\mathrm{y}$, posteriormente, Escalante (1969) dio noticia de ella en el municipio de Tuxtla Chico. Schuman (1969) estudió el cercano tuzanteco; por el lado de las poblaciones fronterizas hubo presencia k'iche' según apunta el Popol Vuh (Recinos, 1957: 79; Carmack, 1979: 119), y un libro de bautismos de la parroquia de Tuxtla Chico registra buena cantidad de apellidos en dicha lengua (Navarrete, 1970: 32).

También hubo presencia "mexicana", toda vez que el imperio mexica conquistó el Soconusco (Vivó, 1942: 125), convirtiéndolo en tributario de Tenochtitlán según consigna el Códice Mendocino (Corona, 1964: 102-103), resguardado por una guarnición militar en Huehuetán. En este lugar, Bruce y Robles (1969) rescataron el vocabulario de una variante nahuat, a la que nombraron "el lenguaje de los ancianos".

Si en la red de rutas de comunicación el Soconusco era un paso natural obligado de los pochteca mexicas, en cuya actividad comercial se mezclaban productos e ideas, materiales suntuarios y modas, y también entrelaces idiomáticos en una región al parecer plurilinguística (Navarrete, 1999), quizá sean éstas las causas de algunas formas estilísticas que tiran hacia otros lugares de origen, presentes en los materiales de la unidad La Chincúa-Plan de la Mesita. 


\section{Bibliografía}

Bruce, Roberto D. y Carlos Robles Uribe

1969 "La lengua de Huehuetán (Waliwi)", Anales del Instituto Nacional de Antropología e Historia, $7^{\text {a }}$ época, I: 115-122.

Carmack, Robert M.

1979 Evolución del reino Quiché. Guatemala: Biblioteca Centroamericana de Ciencias Sociales.

Chapman, Ann

1959 Puertos de intercambio en Mesoamérica prehispánica. México: Instituto Nacional de Antropología e Historia (Serie Historia, III).

Ciudad Real, Antonio

1976 Tratado curioso y docto de las grandezas de la Nueva España, tomo I. México: Universidad Nacional Autónoma de México, Instituto de Investigaciones Históricas.

Coe, Michael D.

1961 La Victoria: An Early Site on the Pacific Coast of Guatemala. Cambridge: Harvard University (Papers of the Peabody Museum of Archaeology and Ethnology, 53).

Cordry, Donald B. y Dorothy M. Cordry

1988 Trajes y tejidos de los indios zoques de Chiapas, México. Tuxtla Gutiérrez: Gobierno del Estado de Chiapas.

Corona Núñez, José (estudio e interpretación)

1964 "Códice Mendocino", Antigüedades de México. Basadas en la recopilación de Lord Kingsborough, tomo I, pp. 1-149. México: Secretaría de Hacienda y Crédito Público.

Cossich, Margarita, Federico Paredes, Byron Hernández, Carol Roldán y Emanuel Serech 2015 'Evidencia del signo 'estrella' en Santa Rosa: implicaciones para el modelo regional de dispersión de Cotzumalguapa”, XXVIII Simposio de Investigaciones Arqueológicas en Guatemala, 2014, pp. 1221-1230, B. Arroyo, L. Méndez y L. Paiz (eds.). Guatemala: Museo Nacional de Arqueología y Etnología.

Culebro, Alberto

1957 Historia de Chiapas: la zona costera del Soconusco a través de la historia. Huixtla: Congreso del Estado de Chiapas.

Domenici, Davide

2009 "Arqueología de la Selva del Ocote, Chiapas", Mundos zoque y maya, pp. 3233, Piero Gorza, Davide Dominici y Claudia Avitable (coords.). Mérida: Universidad Nacional Autónoma de México, Centro Peninsular en Humanidades y Ciencias Sociales (Serie Antologías, 2). 
Escalante, Roberto

1969 "Noticia del mame de Tuxtla Chico", Anales del Instituto Nacional de Antropología e Historia, $7^{\text {a }}$ época, 1: 149-136.

García Vargas, María

1975 “Técnicas textiles de San Bernardino Contla”, Boletín del Departamento de Investigación de las Tradiciones Populares, 2: 79-100.

González Casanova, Pablo

1927 "El Tapachulteco no. 2 sin relación conocida”, Revista Mexicana de Estudios Históricos, 1 (2): 18-26.

Landa, María Elena

1988 La Garrafa. Puebla: Gobierno del Estado de Puebla, Instituto Nacional de Antropología e Historia.

Linares, Eliseo y Carlos Silva Rhoads

2001 "El Tapesco del Diablo y el Castillo, dos cuevas arqueológicas en el cañón del Río de La Venta, Chiapas”, Pueblos y Fronteras, 2: 157-172.

López Vasallo, Ricardo

2012 "La batalla de La Chincúa: la insurgencia mexicana llegó a Chiapas el 12 de abril de 1813", Ateneo, 2a época, 8: 101-134.

Lowe, Gareth, Thomas A. Lee y Eduardo Martínez Espinosa

2000 Izapa: una introducción a las ruinas y monumentos. Tuxtla Gutiérrez: Fundación Arqueológica del Nuevo Mundo, Consejo Estatal para la Cultura y las Artes de Chiapas.

Mastache, Alba Patricia

2005 "El tejido en el México Antiguo", Textiles de México, ayer y hoy. Arqueología Mexicana. Edición especial, 19: 20-31.

Navarrete Cáceres, Carlos

1966 The Sculptural Complex at Cerro Bernal on the Coast of Chiapas. Provo: Brigham Young University (Notes of the New World Archaeological Foundation, 1).

1970 "Evidencia de la lengua quiché en el Soconusco", Boletín de Escritura Maya, 11 (2): 32-33.

1991 “Anotaciones a temas no resueltos: Votán, las 'Columnas de Ben’ y las rocasestelas en el sur de Mesoamérica", Anales de la Academia de Geografía e Historia de Guatemala, LXV: 9-55.

1999 "Influencias mexicanas en el área maya meridional en el Postclásico Tardío: una revisión arqueológica”, Historia General de Guatemala. Época Precolombina, I: 397-416. Guatemala: Asociación de Amigos del País, Fundación para la Cultura y el Desarrollo.

O’Neale, Lila M.

1942 "Early Textiles from Chiapas, Mexico", Middle American Research Records, I (1): $1-6$. 
Pomar, María Teresa

2005 "La indumentaria indígena”, Arqueología Mexicana. Edición especial, 19: 32-79.

Proskouriakoff, Tatiana

1963 Álbum de arquitectura Maya. México: Fondo de Cultura Económica.

Recinos, Adrián

1957 Crónicas indígenas de Guatemala. Guatemala: Universidad de San Carlos.

Ryesky, Diana

1975 "Aspectos de la producción de textiles en telar de cintura en la costa de Oaxaca", Boletín del Departamento de Investigación de las Tradiciones Populares, 2: 101-104.

Schuman, Otto

1969 "El tuzanteco y su posición dentro de la familia mayance", Anales del Instituto Nacional de Antropología e Historia, 7a época, 1: 139-148.

Silva Rhoads, Carlos y Eliseo Linares Villanueva

1993 "El Tapesco del Diablo", Arqueología Mexicana, 1 (3): 76-78.

Stone, Andrea y Sergio Ericastilla Godoy

1999 "Registro de arte rupestre en las Tierras Altas de Guatemala: resultados del reconocimiento de 1997", XII Simposio de Investigaciones Arqueológicas en Guatemala, 1998, pp. 682-695, Juan Pedro Laporte y Héctor Escobedo (eds.). Guatemala: Museo Nacional de Arqueología y Etnología.

Stromsvik, Gustavo

1949 "Las ruinas de Asunción Mita. Informe de su reconocimiento", Antropología e Historia de Guatemala, 1 (2): 23-29.

Thompson, J. Eric S.

1948 "An Archaeological Reconnaissance in the Cotzumalhuapa Region, Escuintla, Guatemala”, Contributions to American Anthropology and Historia, 574 (44): 1-92. Washington: Carnegie Institution.

Vivó, Jorge A.

1942 "Geografía lingüística y política prehispánica de Chiapas y secuencia histórica de sus pobladores”, Revista Geográfica, 2 (4/5/6): 121-156.

Wauchope, Rubert

1942 "Notes on the Age of the Cieneguilla Cave Textiles from Chiapas", Middle American Research Records, 1 (2): 7-8.

Weitlaner Johnson, Irmgard

1954 "Chiptic Cave Textiles from Chiapas", Journal de la Societé des Americanistes, XLIII: 137-147.

1959 "Hilado y tejido", Esplendor del México antiguo, Carmen Cook de Leonard 
(ed.), tomo 1, pp. 439-441. México: Centro de Investigaciones Antropológicas.

2005 “El vestido prehispánico del México antiguo”, Textiles del México de ayer y hoy. Arqueología Mexicana. Edición especial, 19: 8-9.

Wonderly, William L.

1949 "Some Zoquean Phonemic and Morphophonemic Correspondences", International Journal of American Linguistics, XV (1): 1-11.

Carlos Navarrete Cáceres. Guatemalteco. Doctor Honoris Causa por la Universidad Nacional Autónoma de México. Se desempeña como investigador en el Instituto de Investigaciones Antropológicas de esta universidad. Sus especialidades son la arqueología de Tierras Altas del área maya y los Cristos Negros mesoamericanos. Actualmente realiza investigaciones arqueológicas en los Altos Orientales de Chiapas, en los municipios de Trinitaria y Comitán, con el sitio de Chinkultic como punto central de caminos, además de dar seguimiento a la devoción popular del Cristo de Esquipulas en América Latina; recientemente cubrió la Fiesta Grande de Chiapa de Corzo. Entre sus últimas publicaciones pueden mencionarse "Reflexiones arqueológicas a partir de un libro: esculturas de Tajumulco y el Soconusco, Guatemala y Chiapas”, "De las deidades oscuras prehispánicas a los Cristos Negros mesoamericanos", "Lo de Bran, un sitio arqueológico desaparecido del valle de Guatemala y dos braseros de estilo teotihuacano".

cnavarrete@gmail.com 